\title{
Validation of administrative health data for the pediatric population: a scoping review
}

Natalie J Shiff ${ }^{*+}$, Sadia Jama ${ }^{2}$, Catherine Boden ${ }^{3}$ and Lisa M Lix ${ }^{4+}$

\begin{abstract}
Background: The purpose of this research was to perform a scoping review of published literature on the validity of administrative health data for ascertaining health conditions in the pediatric population ( $\leq 20$ years).

Methods: A comprehensive search of OVID Medline (1946 - present), CINAHL (1937 - present) and EMBASE (1947 - present) was conducted. Characteristics of validation studies that were abstracted included the study population, health condition, topic of the validation (e.g., single diagnosis code versus case-finding algorithm), administrative and validation data sources. Inter-rater agreement was measured using Cohen's $\mathrm{K}$. Extracted data were analyzed using descriptive statistics.
\end{abstract}

Results: A total of 37 articles met the study inclusion criteria. Cohen's $k$ for study inclusion/exclusion and data abstraction was 0.88 and 0.97 , respectively. Most studies validated administrative data from the USA (43.2\%) and Canada (24.3\%), and focused on inpatient records (67.6\%). Case-finding algorithms (56.7\%) were more frequently validated than diagnoses codes alone (37.8\%). Five conditions were validated in more than one study: diabetes mellitus, inflammatory bowel disease, asthma, rotavirus infection, and tuberculosis.

Conclusions: This scoping review identified a number of gaps in the validation of administrative health data for pediatric populations, including limited investigation of outpatient populations and older pediatric age groups.

\section{Background}

Administrative health data, which are generated through the routine delivery of health care programs [1], are rich sources of population-based information for research about population health and health services. However, these data were not originally intended for research, leading to many questions about their validity for this purpose. In particular, the use of diagnostic codes in these data, which are typically recorded using the World Health Organization's International Classification of Diseases (ICD), to accurately identify patient populations with acute or chronic diseases has been the focus of multiple validation studies. These studies compare individual diagnostic codes or more complex case-finding algorithms based on combinations of diagnosis codes and other criteria in administrative health data to an external data source, such as survey data, medical charts, or

\footnotetext{
* Correspondence: natalie.shiff@usask.ca

${ }^{\dagger}$ Equal contributors

${ }^{1}$ Department of Pediatrics, College of Medicine, University of Saskatchewan, 103 Hospital Drive, SK S7N OW8, Saskatoon, Canada

Full list of author information is available at the end of the article
}

laboratory test results $[2,3]$. Validation studies and systematic reviews of validation studies [4-6] have primarily focused on adult populations; there have been few validation studies conducted in pediatric populations. A recent review of the quality of validation studies underscored the importance of population-specific studies, because validity may be heterogeneous across populations [7].

Age may be particularly important in the assessment of diagnostic validity because pediatric and adult diseases often differ [7]. For example, only five percent of pediatric patients with juvenile idiopathic arthritis (formerly called juvenile rheumatoid arthritis) have a disease pattern similar to the pattern observed in adult patients with rheumatoid arthritis [8], which may result in discrepant diagnostic validity estimates between the two populations.

The purpose of this study was to synthesize the published literature on the validity of diagnoses recorded in administrative health data for the pediatric population ( $\leq 20$ years). This study was conducted to identify gaps in the literature and opportunities for future research.

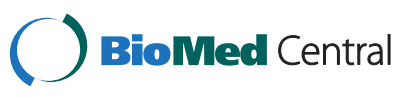

(c) 2014 Shiff et al.; licensee BioMed Central Ltd. This is an Open Access article distributed under the terms of the Creative Commons Attribution License (http://creativecommons.org/licenses/by/2.0), which permits unrestricted use, distribution, and reproduction in any medium, provided the original work is properly credited. 


\section{Methods}

\section{Type of study}

Given that we did not identify any previous syntheses of diagnostic validation studies for administrative health data in the pediatric population, we conducted a scoping review, which is intended to: (a) map an area of study, (b) identify whether a full systematic review of the literature is warranted, (c) summarize and disseminate research, and (d) identify gaps in the literature $[9,10]$. The primary difference between a systematic review and a scoping review is that in the latter, study quality is not the focus of the evaluation $[9,10]$, but in the former it is.

\section{Literature search}

The literature searches were conducted on October 22, 2012. The following electronic databases were comprehensively searched: OVID Medline (1946 - present), CINAHL (1937 - present) and EMBASE (1947 - present). These databases have been used in other systematic reviews of validation studies about diagnostic codes in administrative health databases [7]. Medline is a major bibliographic database for clinical medicine and has its origins in North America. CINAHL primarily indexes the nursing and allied health journals, and includes mainly North American journals as well as some European, Asian, and Australasian journals. EMBASE is a major biomedical and pharmaceutical database that indexes international journals not represented in Medline or CINAHL.

Three conceptual groupings of terms were used to define the scope of this review: (a) validation study, (b) pediatric population and (c) administrative health data. A validation study can be characterized by its research method and outcome measures of sensitivity, specificity, predictive value and receiver operating characteristics. Administrative data include admissions records, discharge data/records/claims/ abstracts, hospital records, outpatient records, inpatient records, physician claims, billing data and medical record linkage. Pediatric populations can be identified by age group (e.g., infant, child, adolescent) and pediatrics specialty.

A preliminary search of the published literature was conducted and the words in the title, abstract, and subject heading were used to develop the final search strategy. This strategy was developed for Medline first (Table 1), and then adapted for EMBASE and CINAHL. Key words

Table 1 Medline search strategy for scoping review

\begin{tabular}{|c|c|c|}
\hline 'Validation study' search terms & 'Pediatric population' search terms & 'Administrative data' search terms \\
\hline 1. validation studies $[\mathrm{MeSH}]$ & 7. exp pediatrics [MeSH] & 10. medical record linkage $[\mathrm{MeSH}]$ \\
\hline 2. case definition*.mp. & \multirow{2}{*}{$\begin{array}{l}\text { 8. *adolescent [MeSH] or exp child } \\
\text { [MeSH] or exp infant [MeSH] }\end{array}$} & 11. "discharge claim*".ab, ti. \\
\hline 3. case validation.mp. & & 12. discharge data.ab, ti. \\
\hline \multirow{2}{*}{$\begin{array}{l}\text { 4. "sensitivity and specificity" }[\mathrm{MeSH}] \text { or } \\
\text { "predictive value of tests" }[\mathrm{MeSH}] \text { or } \\
\text { roc curve }[\mathrm{MeSH}]\end{array}$} & \multirow[t]{3}{*}{9.7 or 8} & 13. "administrative data*".ab, ti. \\
\hline & & 14. "hospital record*.ab, ti. \\
\hline 5. valid*.ab, ti. & & 15. "outpatient record*".ab, ti. \\
\hline \multirow[t]{14}{*}{ 6. or/1-5 } & & 16. "inpatient record*".ab, ti. \\
\hline & & 17. "physician claim*".ab, ti. \\
\hline & & 18. "Clinical Coding" [MeSH] \\
\hline & & $\begin{array}{l}\text { 19. "International Classification of Diseases" [MeSH] } \\
\text { with/sn [Statistics \& Numerical Data] subheading }\end{array}$ \\
\hline & & 20. (ICD9 or "ICD 9" or ICD-9).ab, ti. \\
\hline & & 21. (ICD10 or "ICD 10" or ICD-10).ab, ti. \\
\hline & & 22. "administrative billing code*".ab, ti. \\
\hline & & 23. hospital-discharge data.ab, ti. \\
\hline & & 24. hospital billing data.ab, ti. \\
\hline & & 25. "discharge code*".ab, ti. \\
\hline & & 26. "admissions record*".ab, ti. \\
\hline & & 27. "discharge record*.ab, ti. \\
\hline & & 28. "discharge abstract*".ab, ti. \\
\hline & & 29. or/10-28 \\
\hline
\end{tabular}


and subject headings were combined using Boolean operators. No limits were placed on publication date or type (e.g., journal article, systematic review). The reference lists of all included articles were examined to identify additional articles that may have been missed during the database search.

The bibliographic information (e.g., title, authors, abstract, subject headings, and website address [where applicable]) was imported into Refworks bibliographic management software for storage and removal of duplicate citations (http://www.refworks.com/).

\section{Selection and data extraction}

Following the removal of duplicate citations, a training phase was used to ensure that study inclusion criteria were consistently applied for a randomly selected subset of approximately $5 \%$ of the studies. A citation was included if: (a) analyses were conducted for patients aged 0 to 20 years of age, (b) results of primary research were reported in peer reviewed publications, (c) it was published in English as translation resources were not available, and (d) it was a validation study of administrative health data. Administrative health data differ from registries in that the latter refer to data systems in which information about all cases of a specified disease in a given population are recorded [11]. Examples include cancer registries, birth defect registries, and twin registries. Studies about the validity of registries were not included in the scoping review.

Following the training phase, two authors (NS and SJ) applied the study inclusion criteria to another randomly selected sample of 23 studies, and kappa was calculated for the decision to include or exclude (yes or no). Both authors extracted data from this validation set using a standardized form. All data extracted by each of the respective authors were then coded and pooled, and kappa was calculated for the pooled results of the data extraction. Subsequently, one investigator (SJ) applied the inclusion criteria to all remaining studies and extracted data from the retained studies.

The abstracted information included characteristics of the citation (e.g., publication year), study population (e.g., country of origin, age group and gender), health condition(s) that were investigated, administrative health data (e.g., the diagnosis codes or case finding algorithms that were validated, type of data source, type of diagnostic coding system), and the external data used to conduct the validation.

\section{Statistical analyses}

Inter-rater agreement was assessed using Cohen's $\kappa$ [12] for: (a) study inclusion and (b) data extraction. As well, 95\% confidence intervals (CIs) were calculated. The data were analyzed using descriptive statistics, including frequencies and percentages.

\section{Results}

A total of 1204 abstracts were identified by the literature search (Figure 1). After removing duplicates, 817 unique abstracts were screened for study inclusion. Fifteen were excluded based only on the title and abstract (1.8\%). Thus, a total of 802 articles (98.2\%) underwent full text review. Of this number, 765 (95.4\%) were excluded for the following reasons (reasons are not mutually exclusive): 608 (75.8\%) were not validation studies, 466 (58.1\%) did not use administrative health data, and 216 (26.9\%) did not conduct separate validation analyses for pediatric patients. Thirty-six articles met criteria for further analysis. A hand search of the reference lists of included studies identified one additional article, yielding a final sample of 37 articles. Cohen's $\kappa$ for study inclusion/exclusion and data abstraction was 0.88 (95\% CI $0.72,1.00)$ and 0.97 (95\% CI 0.94, 0.99), respectively.

The characteristics of included studies are summarized in Table 2. Increasing numbers of validation studies were published over time, with 11 (29.7\%) published between 2006 and 2010 and a further 12 (32.4\%) identified between 2011 and 2012 (up to the end of the search period). Just over $40 \%$ of the studies (16 studies, $43.2 \%$ ) were conducted using administrative health data from the United States, followed by Canada (9 studies, 24.3\%). All validation studies included both males and females. There was a trend of smaller numbers of validation studies as age increased, with fewer studies (17 studies, 45.9\%) including individuals aged 16 to 20 years.

Slightly more than one-third of studies (14 studies, $37.8 \%$ ) validated diagnosis codes, while more than half evaluated case-finding algorithms (21 studies, 56.8\%), which use a combination of diagnosis codes and other criteria (e.g., procedure codes) to identify cases with the condition of interest. Two studies (5.4\%) validated both diagnosis codes and case-finding algorithms.

Only five conditions were investigated in more than one study: diabetes (10.6\%) [13-16], inflammatory bowel disease $(5.4 \%)[17,18]$, asthma $(5.4 \%)[19,20]$, rotavirus infection (5.4\%) [21,22], and tuberculosis $(5.4 \%)$ [23,24]. However, a diverse range of conditions were investigated in single studies, including obesity [25], vaccine-related illness [26], injuries [27], autism [28], febrile neutropenia in oncology patients [29], high risk conditions [30], dermatologic conditions [31-33], congenital anomalies [34], cardiac defects [35], respiratory illnesses excluding asthma [36-38], neurologic conditions [39], other gastrointestinal conditions [40-43], genitourinary conditions [44,45], serum sickness [46], thrombosis [47], maternal/perinatal conditions [48], and drug-related anaphylaxis [49].

Administrative health data sources that were validated consisted of inpatient (25 studies, $67.6 \%$ ), outpatient (9 studies, $24.3 \%$ ), and emergency room records (2 studies, 


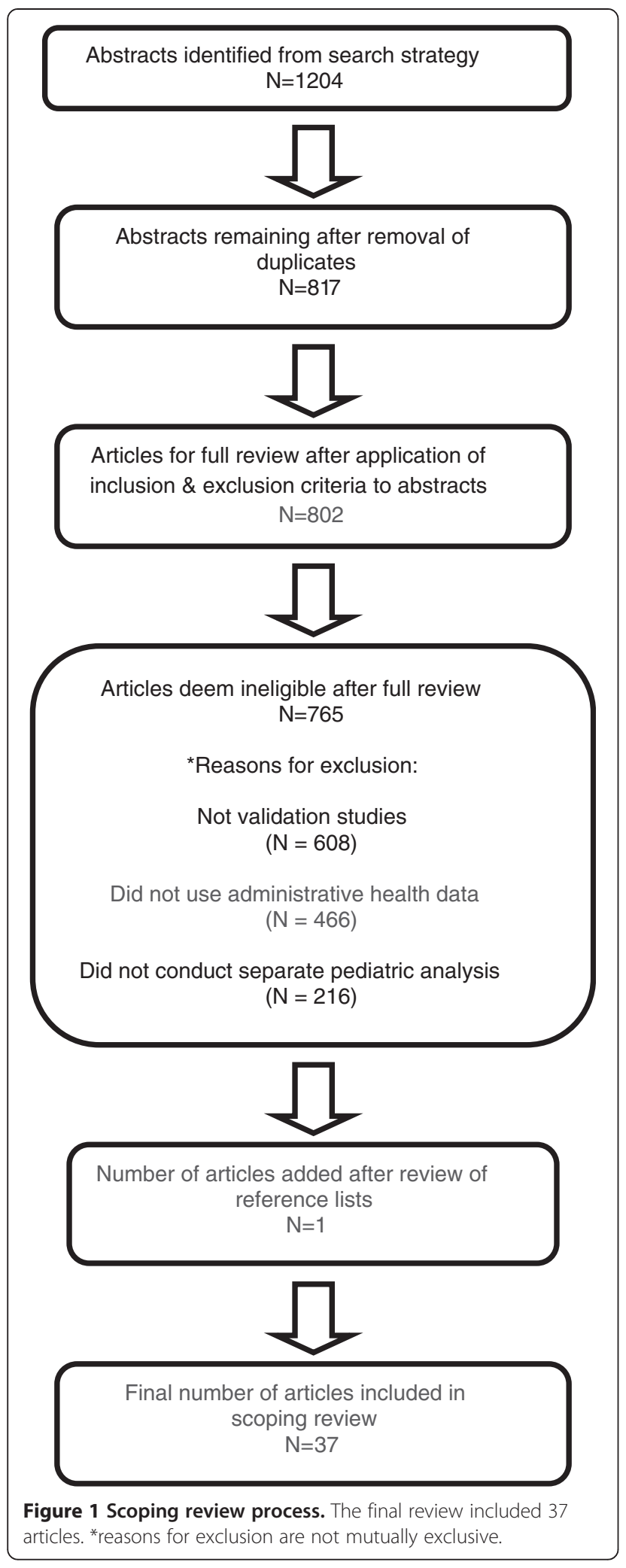

5.4\%), as well as pharmacy data (2 studies, 5.4\%). Studies that validated individual diagnoses or case-finding algorithms in a single database were most frequent (25 studies,
$67.6 \%$ ). Eighty percent (20 studies) of the 25 studies that validated data from a single administrative database used inpatient administrative data (54.1\% of 37 studies), followed by outpatient administrative data (4 studies, $10.8 \%$ of 37 studies) and emergency department data ( 1 study, $2.7 \%$ of 37 studies). Twelve studies $(32.4 \%$ of 37 studies) validated information from multiple, linked administrative data sources; five of these studies included inpatient records (13.5\% of 37 studies) and five included outpatient records as one of the databases.

The main diagnosis coding systems that were validated included ICD-9 or ICD-9 CM (28 studies, 75.7\%) and ICD-10 or ICD-10-CA (8 studies, 21.6\%). The most frequent external data sources used to validate administrative data were medical charts (23 studies, $62.2 \%$ ) and diseasespecific registry data (6 studies, $16.2 \%$ ). Other validation sources used included clinical databases, laboratory records, and survey data. Validation measures reported include sensitivity (24 studies, 64.8\%), specificity (20 studies, $54.0 \%$ ), positive predictive value (17 studies, $45.9 \%$ ), and negative predictive value (8 studies, $21.6 \%$ ).

\section{Discussion}

The prevalence of chronic pediatric conditions has increased over recent decades; it is estimated that between $16 \%$ and $51 \%$ of children have at least one chronic condition [50-52]. Medical advances have improved survival for conditions that were once fatal, resulting in an increasing number of children with special healthcare needs [50-52]. In order to allow for resource planning and optimization of care, the long-term outcomes of children and youth with chronic conditions need to be determined, as does their healthcare utilization [52]. Administrative health data are an appropriate source to conduct long-term follow-up studies, but validation studies are important to ensure that true cases of disease can be ascertained in these data.

To the best of our knowledge, this is the first scoping review to describe validation studies of administrative health data in the pediatric population. Only a small number of pediatric validation studies were identified, whereas a recent systematic review about the quality of reporting of administrative data validation studies that included all age groups, identified 271 studies published prior to June 2009 [7], most of which focused on the adult population. The increasing number of pediatric validation studies in recent years suggests that there is growing recognition that pediatric populations are important to consider separately from adult populations when validating administrative health data. The vast majority of studies were conducted in North America, reflecting a general trend for validation studies [4-6]. Most of the 37 studies included in this scoping review validated complex case-finding algorithms that use a 
Table 2 Characteristics of validation studies in the scoping review

\begin{tabular}{lc}
\hline Study characteristic $(\boldsymbol{N}=\mathbf{3 7})$ & $\boldsymbol{n}(\%)$ \\
\hline Year of publication & $4(10.8)$ \\
Prior to 2001 & $10(27.0)$ \\
$2001-2005$ & $11(29.7)$ \\
$2006-2010$ & $12(32.4)$ \\
$2011-2012$ & \\
Country of origin & $16(43.2)$ \\
USA & $9(24.3)$ \\
Canada & $3(8.1)$ \\
Denmark & $3(8.1)$ \\
New Zealand & $2(5.4)$ \\
China & $2(5.4)$ \\
United Kingdom & $2(5.4)$ \\
Other &
\end{tabular}

Age group (years) ${ }^{\mathrm{a}}$

$0-5$

$6-10$

$11-15$

$16-20$

\section{Condition validated}

Diabetes

Inflammatory bowel disease

Asthma

Rotavirus infection

Tuberculosis

Other

Topic of validation

Diagnosis codes only

Case-finding algorithm

Both

Administrative data source

Inpatient

Outpatient

Emergency department

Pharmacy

Diagnosis coding system ${ }^{a}$

ICD-9 or ICD-9-CM

ICD-10 or ICD-10-CA

Other

Validation data source ${ }^{a}$

Medical chart

Disease registry

Clinical database $8(21.6)$
Table 2 Characteristics of validation studies in the scoping review (Continued)

\begin{tabular}{lc}
\hline Laboratory data & $4(10.8)$ \\
Survey & $2(5.4)$ \\
Validation measure $^{\text {a }}$ & \\
Sensitivity & $24(64.8)$ \\
Specificity & $20(54.0)$ \\
PPV $^{b}$ & $17(45.9)$ \\
NPV & $8(21.6)$ \\
Other $^{d}$ & $4(10.8)$ \\
\hline
\end{tabular}

${ }^{\mathrm{a} C a t e g o r i e s}$ are not mutually exclusive; ${ }^{\mathrm{b}} P P V=$ positive predictive value; ${ }^{C} N P V=$ negative predictive value; ${ }^{d}$ Other $=$ correlation coefficient, relative risk of association, kappa, percentage of agreement.

variety of information found in administrative health data to ascertain disease cases.

Case-finding algorithms typically take advantage of linked administrative health databases, whereas validation of individual diagnoses may only take place in a single administrative data source. In many administrative data systems, data linkage creates the opportunity to evaluate case-finding algorithms that will have sensitivity or specificity that is greater than what can be observed by examining a diagnosis in an unlinked database.

It is surprising that no validation studies were identified for common chronic pediatric conditions such as attention deficit/hyperactivity disorder and obesity [50]. Only diabetes, inflammatory bowel disease, asthma, tuberculosis, and rotavirus infection were validated in more than one setting. It is well known that diabetes can be ascertained from administrative health data with high specificity and sensitivity for adult populations, which may have contributed to increased interest in performing validation studies for this diagnosis in the pediatric population. In addition, with the growth in rates of juvenile diabetes, this is an important condition for chronic disease research and surveillance [50]. There is a gap in the literature for conditions validated in the adults but not in the pediatric setting. Rheumatoid arthritis is one example of a chronic condition for which several validation studies have been published in the adult population [53-55] but similar validation studies are lacking in the pediatric age group. In fact, no validation studies were found for chronic in28 (75.7) flammatory arthritis in the pediatric population at the time of this scoping review.

While this scoping review has several strengths, including the breadth of citation databases investigated, the multiple health conditions that were included, and the range of characteristics of the studies that were examined, it does have some limitations. Only English language publications were included. Conference proceedings and articles that were not published in peer-review journals were excluded. Publication bias may affect the generalizability 
of the scoping review results. Nevertheless, these factors taken together are not likely to result in a large number of missing research studies, and hence cannot account for the relative dearth of pediatric administrative data validation studies that were identified.

For conditions with several published validation studies in the pediatric population, such as diabetes, disease specific systematic reviews evaluating the quality of studies should be examined, but only once more studies have been published; at present, there are too few validation studies in pediatric publications to warrant systematic reviews. Many pediatric conditions are treated primarily in an outpatient setting, and almost all chronic diseases in this population require at least some outpatient care, yet validation studies in this setting are lacking. Patients with milder disease or better access to outpatient-based services may never need hospitalization, and validation studies primarily based on inpatient data likely do not capture the true spectrum of chronic disease severity. Validation studies in the outpatient setting can be challenging to conduct due to small patient numbers in individual centres, lack of standardized charting, and difficulties accessing medical records. As electronic medical records become more widely available, this could potentially facilitate validation studies in the outpatient setting.

\section{Conclusions}

Numerous studies about the diagnostic validity of administrative health data for the adult population have been published [7], but studies about the pediatric population have been limited in number and scope, despite the fact that diagnoses may not be equally valid in both populations. An increasing number of children are living with chronic conditions. Administrative health data can be used to estimate the burden of these conditions and provide long-term outcomes data for studies about mortality, health care utilization, and comorbid conditions. In order for administrative data to serve these purposes, their validity must be established. Our scoping review of published literature on diagnostic validity of administrative health data in the pediatric population revealed multiple gaps in the pediatric literature. Common chronic pediatric conditions have not been validated in a multiple settings, the number of validation studies decreased with increasing age within the pediatric population, and although many pediatric conditions are treated primarily in an outpatient setting, validation studies in this setting are lacking. Further studies are needed to examine validity for a broad spectrum of pediatric health conditions, in outpatient populations, and in both younger and older age groups.

\section{Competing interests}

The authors declare that they do not have any competing interests.

\section{Authors' contributions}

NJS, LML, CB conceived the study and participated in its design. CB developed and conducted the literature search strategy. NJS and SJ conducted the data extraction. NJS, SJ, and LML carried out the statistical analyses. NJS, CB, and LML drafted the manuscript. All authors read and approved the final manuscript.

\section{Acknowledgements}

This research was supported by funding from the Canadian Arthritis Network Rapid Impact Platform Program, the Saskatchewan Health Research Foundation, and the Centennial Chair Program at the University of Saskatchewan.

\section{Author details}

${ }^{1}$ Department of Pediatrics, College of Medicine, University of Saskatchewan, 103 Hospital Drive, SK S7N 0W8, Saskatoon, Canada. ${ }^{2}$ Department of Community Health and Epidemiology, College of Medicine, University of Saskatchewan, 103 Hospital Drive, SK S7N 0W8, Saskatoon, Canada.

${ }^{3}$ University Library, University of Saskatchewan, Room 1441, Leslie and Irene Dube Health Sciences Library, 104 Clinic Place, SK S7N 5E5, Saskatoon, Canada. ${ }^{4}$ Department of Community Health Sciences, S113-750 Bannatyne Avenue, University of Manitoba, MB R3E 0W3, Winnipeg, Canada.

Received: 21 May 2013 Accepted: 15 May 2014

Published: 22 May 2014

\section{References}

1. Virnig BA, McBean M: Administrative data for public health surveillance and planning. Annu Rev Public Health 2001, 22(1):213-230.

2. van Walraven $C$, Austin P: Administrative database research has unique characteristics that can risk biased results. J Clin Epidemio/ 2012, 65(2):126-131.

3. Van Walraven C, Bennett C, Forster AJ: Administrative database research infrequently used validated diagnostic or procedural codes. J Clin Epidemiol 2011, 64(10):1054-1059.

4. Quach S, Blais C, Quan H: Administrative data have high variation in validity for recording heart failure. Can J Cardiol 2010, 26(8):306-312.

5. Sharabiani MT, Aylin P, Bottle A: Systematic review of comorbidity indices for administrative data. Med Care 2012, 50(12):1109-1118.

6. Vlasschaert ME, Bejaimal SA, Hackam DG, Quinn R, Cuerden MS, Oliver MJ, lansavichus A, Sultan N, Mills A, Garg AX: Validity of administrative database coding for kidney disease: a systematic review. Am J Kidney Dis 2011, 57(1):29-43.

7. Benchimol El, Manuel DG, To T, Griffiths AM, Rabeneck L, Guttmann A: Development and use of reporting guidelines for assessing the quality of validation studies of health administrative data. J Clin Epidemiol 2011, 64(8):821-829.

8. Cassidy JT: Textbook of Pediatric Rheumatology. 6th edition. Philadelphia, PA: Saunders; 2011.

9. Arksey H, O'Malley L: Scoping studies: towards a methodological framework. Int J Soc Res Methodol 2005, 8(1):19-32.

10. Levac D, Colquhoun H, O'Brien KK: Scoping studies: advancing the methodology. Implement Sci 2010, 5(1):69.

11. Porta MS: International Epidemiological Association: A Dictionary of Epidemiology. 5th edition. Oxford; New York: Oxford University Press; 2008.

12. Cohen J: A coefficient of agreement for nominal scales. Educ Psychol Meas 1960, 20(1):37-46.

13. Hodgson S, Beale L, Parslow RC, Feltbower RG, Jarup L: Creating a national register of childhood type 1 diabetes using routinely collected hospital data. Pediatr Diabetes 2012, 13(3):235-243.

14. Amed S, Vanderloo SE, Metzger D, Collet JP, Reimer K, Mccrea P, Johnson $J A$ : Validation of diabetes case definitions using administrative claims data. Diabetic Med 2011, 28(4):424-427.

15. Dart AB, Martens PJ, Sellers EA, Brownell MD, Rigatto C, Dean HJ: Validation of a pediatric diabetes case definition using administrative health data in Manitoba. Canada Diabetes Care 2011, 34(4):898-903.

16. Guttmann A, Nakhla M, Henderson M, To T, Daneman D, Cauch-Dudek K, Wang X, Lam K, Hux J: Validation of a health administrative data algorithm for assessing the epidemiology of diabetes in Canadian children. Pediatr Diabetes 2010, 11(2):122-128. 
17. Liu L, Allison JE, Herrinton LJ: Validity of computerized diagnoses, procedures, and drugs for inflammatory bowel disease in a northern California managed care organization. Pharmacoepidemiol Drug Saf 2009 18(11):1086-1093.

18. Benchimol El, Guttmann A, Griffiths AM, Rabeneck L, Mack DR, Brill H, Howard J, Guan J, To T: Increasing incidence of paediatric inflammatory bowel disease in Ontario, Canada: Evidence from health administrative data. Gut 2009, 58(11):1490-1497.

19. Moth G, Vedsted P, Schiotz PO: National registry diagnoses agree with medical records on hospitalized asthmatic children. Acta Paediatr 2007, 96(10):1470-1473.

20. To T, Dell S, Dick PT, Cicutto L, Harris JK, MacLusky IB, Tassoudji M: Case verification of children with asthma in Ontario. Pediatr Allergy Immuno 2006, 17(1):69-76.

21. Hsu VP, Staat MA, Roberts N, Thieman C, Bernstein DI, Bresee J, Glass RI, Parashar UD: Use of active surveillance to validate International Classification of Diseases code estimates of rotavirus hospitalizations in children. Pediatrics 2005, 115(1):78-82

22. Matson DO, Estes MK: Impact of rotavirus infection at a large pediatric hospital. J Infect Dis 1990, 162(3):598-604.

23. Siegel D, Song X, Klontz K, Pastor W, Singh N: Epidemiology of childhood tuberculosis: use and evaluation of the pediatric health information system to assess local and national incidence. Pediatr Infect Dis J 2011, 30(5):428-430

24. Trepka MJ, Beyer TO, Proctor ME, Davis JP: An evaluation of the completeness of tuberculosis case reporting using hospital billing and laboratory data; Wisconsin, 1995. Ann Epidemiol 1999, 9(7):419-423.

25. Kuhle S, Kirk SF, Ohinmaa A, Veugelers PJ: Comparison of ICD code-based diagnosis of obesity with measured obesity in children and the implications for health care cost estimates. BMC Med Res Methodol 2011, 11:173.

26. Nash JQ, Chandrakumar M, Farrington CP, Williamson S, Miller E: Feasibility study for identifying adverse events attributable to vaccination by record linkage. Epidemiol Infect 1995, 114(3):475-480.

27. Kostylova A, Swaine B, Feldman D: Concordance between childhood injury diagnoses from two sources: An injury surveillance system and a physician billing claims database. Inj Prev 2005, 11(3):186-190.

28. Dodds L, Spencer A, Shea S, Fell D, Armson BA, Allen AC, Bryson S: Validity of autism diagnoses using administrative health data. Chronic Dis Can 2009, 29(3):102-107.

29. Breitfeld PP, Dale T, Kohne J, Hui S, Tierney WM: Accurate case finding using linked electronic clinical and administrative data at a children's hospital. J Clin Epidemiol 2001, 54(10):1037-1045

30. Daley TC, Simeonsson RJ, Carlson E: Constructing and testing a disability index in a US sample of preschoolers with disabilities. Disabil Rehabil 2009, 31(7):538-552.

31. Das DK, Baker MG, Venugopal K: Increasing incidence of necrotizing fasciitis in New Zealand: A nationwide study over the period 1990 to 2006. J Infect 2011, 63(6):429-433.

32. Wu JJ, Black MH, Smith N, Porter AH, Jacobsen SJ, Koebnick C: Low prevalence of psoriasis among children and adolescents in a large multiethnic cohort in southern California. J Am Acad Dermatol 2011, 65(5):957-964

33. O'Sullivan CE, Baker MG: Proposed epidemiological case definition for serious skin infection in children. J Paediatr Child Health 2010, 46(4):176-183.

34. Larsen H, Nielsen GL, Bendsen J, Flint C, Olsen J, Sørensen H: Predictive value and completeness of the registration of congenital abnormalities in three Danish population-based registries. Scand J Public Health 2003, 31(1):12-16.

35. Frohnert BK, Lussky RC, Alms MA, Mendelsohn NJ, Symonik DM, Falken MC Validity of hospital discharge data for identifying infants with cardiac defects. J Perinatol 2005, 25(11):737-742.

36. Beitel AJ, Olson KL, Reis BY, Mandl KD: Use of emergency department chief complaint and diagnostic codes for identifying respiratory illness in a pediatric population. Pediatr Emerg Care 2004, 20(6):355-360.

37. Nelson EA, Tam JS, Yu LM, Li AM, Chan PK, Sung RY: Assessing disease burden of respiratory disorders in Hong Kong children with hospital discharge data and linked laboratory data. Hong Kong Med J 2007, 13(2):114-121.
38. Hamvas A, Kwong P, DeBaun M, Schramm W, Cole FS: Hyaline membrane disease is underreported in a linked birth-infant death certificate database. Am J Public Health 1998, 88(9):1387-1389.

39. Vestergaard M, Obel C, Henriksen TB, Christensen J, Madsen KM, Ostergaard JR, Olsen J: The Danish National Hospital Register is a valuable study base for epidemiologic research in febrile seizures. J Clin Epidemio/ 2006, 59(1):61-66

40. Nelson EAS, Chiu SS, Ip KS, Lau D, Lau YL, Ng CH, Ng DK, Poon KH, Yiu WL, Kwok WK, Tam P, Yeung CK, Tam JS, Bock H, Boudville I, Tang HH: Intussusception trends in Hong Kong children. Hong Kong Med J 2007, 13(4):279-283.

41. Chang S, Begier EM, Schech SD, Venus P, Shatin D, Braun MM, Ball R: Perinatal hepatitis $B$ transmission and vaccination timing in a managed care cohort: assessment of the temporary delay in newborn hepatitis $B$ vaccination due to thimerosal content. Pediatr Infect Dis J 2007 26(4):329-333

42. Shaklee J, Zerr DM, Elward A, Newland J, Leckerman K, Asti L, Guth R, Bass J, Selvarangan R, Coffin S, Zaoutis T: Improving surveillance for pediatric Clostridium difficile infection: Derivation and validation of an accurate case-finding tool. Pediatr Infect Dis J 2011, 30(3):e38-e40.

43. Dydensborg S, Toftedal P, Biaggi M, Lillevang ST, Hansen DG, Husby S: Increasing prevalence of coeliac disease in Denmark: a linkage study combining national registries. Acta Paediatr 2012, 101(2):179-184.

44. Tieder JS, Hall M, Auger KA, Hain PD, Jerardi KE, Myers AL, Rahman SS, Williams DJ, Shah SS: Accuracy of Administrative Billing Codes to Detect Urinary Tract Infection Hospitalizations. Pediatrics 2011, 128(2):323-330.

45. Ong KL, Apostal M, Comstock N, Hurd S, Webb TH, Mickelson S, Scheftel J, Smith G, Shiferaw B, Boothe E, Gould LH: Strategies for surveillance of pediatric hemolytic uremic syndrome: Foodborne Diseases Active Surveillance Network (FoodNet), 2000-2007. Clin Infect Dis 2012, 54(Suppl 5):S424-S431.

46. Heckbert SR, Stryker WS, Coltin KL, Manson JE, Platt R: Serum sickness in children after antibiotic exposure: estimates of occurrence and morbidity in a health maintenance organization population. Am J Epidemiol 1990, 132(2):336-342.

47. Tuckuviene R, Kristensen SR, Helgestad J, Christensen AL, Johnsen SP: Predictive value of pediatric thrombosis diagnoses in the Danish National Patient Registry. Clin Epidemiol 2010, 2(1):107-122.

48. Taylor LK, Travis S, Pym M, Olive E, Henderson-Smart DJ: How useful are hospital morbidity data for monitoring conditions occurring in the perinatal period? Aust N Z J Obstet Gynaecol 2005, 45(1):36-41.

49. West SL, D'Aloisio AA, Ringel-Kulka T, Waller AE, Clayton Bordley W: Population-based drug-related anaphylaxis in children and adolescents captured by South Carolina Emergency Room Hospital Discharge Database (SCERHDD) (2000-2002). Pharmacoepidemiol Drug Saf 2007 16(12):1255-1267.

50. Van Cleave J, Gortmaker SL, Perrin JM: Dynamics of obesity and chronic health conditions among children and youth. JAMA 2010, 303(7):623-630.

51. Halfon N, Newacheck PW: Evolving notions of childhood chronic illness. JAMA 2010, 303(7):665-666.

52. Wise PH: The future pediatrician: the challenge of chronic illness. J Pediatr 2007, 151(5):S6-S10.

53. Kim SY, Servi A, Polinski JM, Mogun H, Weinblatt ME, Katz JN: Validation of rheumatoid arthritis diagnoses in health care utilization data. Arthritis Res Ther 2011, 13:R32.

54. Ng B, Aslam F, Petersen NJ, Yu H, Suarez-almazor ME: Identification of rheumatoid arthritis patients using an administrative database: a Veterans Affairs study. Arthritis Res Ther 2012, 64(10):1490-1496.

55. Singh JA, Holmgren AR, Noorbaloochi S: Accuracy of Veterans Administration databases for a diagnosis of rheumatoid arthritis. Arthritis Rheum 2004, 51(6):952-957.

doi:10.1186/1472-6963-14-236

Cite this article as: Shiff et al:: Validation of administrative health data

for the pediatric population: a scoping review. BMC Health Services Research 2014 14:236. 